VoL. 37 (1988) [113-120]

\title{
A DUAL CHARACTERISATION OF THE EXISTENCE OF SMALL COMBINATIONS OF SLICES
}

\author{
ROBERT DEVILLE
}

\begin{abstract}
We characterise, by a property of roughness, the norms of a Banach space $X$ such that the dual unit ball has no small combination of $w^{\star}$-slices. Among separable Banach spaces, the existence of an equivalent norm for this new property of roughness characterises spaces which contain an isomorphic copy of $\ell_{1}(\mathrm{~N})$.
\end{abstract}

\section{INTRODUCTION}

Throughout this paper, $X$ denotes a Banach space, $B(X)$ its unit ball, $S(X)$ its unit sphere, $B\left(X^{\star}\right)$ the unit ball of its dual and $S\left(X^{\star}\right)$ the unit sphere of its dual. Let us first recall some basic definitions and introduce the notion of "average rough norm":

\section{Definitions.}

1. Let $C$ be a closed convex subset of $X$. We say that $C$ is $\varepsilon$-dentable (respectively $\varepsilon-w^{\star}$-dentable if $X$ is a dual space) if there exists a slice $S$ (respectively $w^{\star}$-slice $S$ ) with $\operatorname{diam}(S)<\varepsilon(\operatorname{diam}(S)$ denotes the diameter of $S)$.

We say that $C$ contains an $\varepsilon$-combination of slices (respectively an $\varepsilon$-combination of $w^{\star}$-slices) if there are slices (respectively $w^{\star}$-slices) $S_{1}, \ldots, S_{n}$ of $C$ with $\operatorname{diam}\left(\frac{1}{n}\left(S_{1}+\cdots+S_{n}\right)\right)<\varepsilon$.

2. A one-sided Gâteaux differential of the norm \|\| of $X$ at $x \in X$ is a function $d^{+}\|x\|: X \rightarrow \mathbb{R}$ such that, for all $u \in X, \quad d^{+}\|x\|(u)=\lim _{t \rightarrow 0^{+}} \frac{\|x+t u\|-\|x\|}{t}$.

3. We say that the norm of $X$, or merely $X$ where there is no ambiguity, is $\varepsilon$-rough if for all $x \in S(X)$ and for all $\eta>0$ there exist $y, z \in S(X)$ and $u \in S(X)$ such that

(a) $\|y-x\|<\eta$ and $\|z-x\|<\eta$
(b) $\left(d^{+}\|y\|-d^{+}\|z\|\right)(u)>\varepsilon-\eta$.

Received 7 April 1987

This research was completed while the author was visiting the University of Alberta. The author wishes to thank Professors N. Tomczak-Jaegermann and V. Zizler for their support and for challenging discussions.

Copyright Clearance Centre, Inc. Serial-fee code: 0004-9729/88 \$A2.00+0.00. 
4. We say that the norm of $X$, or merely $X$ when there is no ambiguity, is $\varepsilon$-average rough if, for all $x_{1}, \ldots, x_{n} \in S(X)$ and for all $\eta>0$, there exist $y_{1}, \ldots, y_{n}, z_{1}, \ldots, z_{n} \in$ $S(X)$ and $u \in S(X)$ such that:

(a) for all $i, 1 \leqslant i \leqslant n, \quad\left\|y_{i}-x_{i}\right\|<\eta$ and $\left\|z_{i}-z_{i}\right\|<\eta$

(b) $\frac{1}{n} \sum_{i=1}^{n}\left(d^{+}\left\|y_{i}\right\|-d^{+}\left\|z_{i}\right\|\right)(u)>\varepsilon-\eta$.

We refer the reader to [2] and [7] for a study of the small combination of slices property.

An obvious observation is that, if $X$ is $\varepsilon$-average rough, then $X$ is $\varepsilon$-rough. More precisely, in the definition of $\varepsilon$-average roughness, for any $n \in \mathrm{N}$ and any $x_{1}, \ldots, x_{n} \in$ $S(X)$, there exists a common direction of roughness $u$ for many of the $x_{i}, 1 \leqslant i \leqslant n$ (but not necessarily for all of them).

In [6], Leach and Whitfield introduced and studied rough norms. In [5], John and Zizler have shown that $X$ is $\varepsilon$-rough if and only if $B\left(X^{\star}\right)$ is not $\varepsilon$-w $w^{\star}$-dentable. Moreover, it is shown, for instance in [3], that for separable Banach spaces, the existence of an equivalent rough norm characterises the separable Banach spaces with non-separable dual.

We shall show that $X$ is $\varepsilon$-average rough if and only if $B\left(X^{\star}\right)$ does not contain any $\varepsilon$-combination of $w^{\star}$-slices. Moreover, for separable Banach spaces, the existence of an equivalent $\varepsilon$-average rough norm, for some $\varepsilon>0$, characterises the spaces which contain $\ell^{1}(\mathrm{~N})$.

\section{Characterisation of aVerage ROUgh NORMS.}

Theorem 1. Let $0<\varepsilon<1$ and let $X$ be a Banach space. The following conditions are equivalent:

1. $X$ is $\varepsilon$-average rough;

2. For each $x_{1}, \ldots, x_{n} \in S(X)$,

$$
\limsup _{\|y\| \rightarrow 0} \frac{1}{n} \sum_{i=1}^{n}\left(\frac{\left\|x_{i}+y\right\|+\left\|x_{i}-y\right\|-2}{\|y\|}\right) \geqslant \varepsilon ;
$$

3. $B\left(X^{*}\right)$ does not contain any $\varepsilon$-combination of $w^{\star}$-slices.

Dually, an analogous result holds:

TheOREM 2. The following conditions are equivalent:

1. $X^{\star}$ is $\varepsilon$-average rough;

2. For each $x_{1}^{\star}, \ldots, x_{n}^{\star} \in S\left(X^{\star}\right)$,

$$
\limsup _{y^{\star} \in X^{\star},\left\|y^{\star}\right\| \rightarrow 0} \frac{1}{n} \sum_{i=1}^{n}\left(\frac{\left\|x_{i}^{\star}+y^{\star}\right\|+\left\|x_{i}^{\star}-y^{\star}\right\|-2}{\left\|y^{\star}\right\|}\right) \geqslant \varepsilon ;
$$


3. $B(X)$ does not contain any $\varepsilon$-combination of slices.

REMARK:: These two results are isometric, and give dual characterisation of the existence of small combinations of slices $B(X)$ (respectively small combinations of $w^{\star}$ slices of $\left.B\left(X^{\star}\right)\right)$.

Proof of ThEOREM 1: Some of the arguments are refinements of ideas in [5] and [8].

(1) $\Rightarrow$ (3): Suppose $X$ is $\varepsilon$-average rough and let $S_{1}, \ldots, S_{n}$ be $w^{\star}$-slices of $B\left(X^{\star}\right)$. Replacing $S_{1}, \ldots, S_{n}$ by smaller $w^{\star}$-slices, we can assume that for all $i \in$ $\{1, \ldots, n\}, S_{i}=S_{i}\left(x_{i}, B\left(X^{\star}\right), \eta\right)=\left\{f \in B\left(X^{\star}\right) ; f\left(x_{i}\right)>1-\eta\right\}$, with $x_{i} \in S(X)$ and $\eta>0$.

By hypothesis there exists $y_{1}, \ldots, y_{n}, z_{1}, \ldots, z_{n} \in S(X)$ and $u \in S(X)$ satisfying:

a. for all $i,\left\|y_{i}-x_{i}\right\|<\eta$ and $\left\|z_{i}-x_{i}\right\|<\eta$;

b. $\frac{1}{n} \sum_{i=1}^{n}\left(d^{+}\left\|y_{i}\right\|-d^{+}\left\|z_{i}\right\|\right)(u)>\varepsilon-\eta$.

Using the Ascoli-Mazur theorem $([4])$, choose $f_{i}, g_{i} \in S\left(X^{\star}\right)$ satisfying $f_{i}\left(y_{i}\right)=1$, $f_{i}(u)=d^{+}\left\|y_{i}\right\|(u), g_{i}(z)=1$ and $g_{i}(u)=d^{+}\left\|z_{i}\right\|(u)$. Condition (b) implies that $\frac{1}{n} \sum_{i=1}^{n}\left(f_{i}-g_{i}\right)(u)>\varepsilon-\eta$ hence $\left\|\frac{1}{n} \sum_{i=1}^{n}\left(f_{i}-g_{i}\right)\right\|>\varepsilon-\eta$. On the other hand, $f_{i}\left(x_{i}\right) \geqslant f_{i}\left(y_{i}\right)-\left\|x_{i}-y_{i}\right\|>1-\eta$ and so $f_{i} \in S_{i}$; an analogous calculation shows that $g_{i} \in S_{i}$ and we have shown that $\operatorname{diam}\left(\frac{1}{n}\left(\sum_{i=1}^{n} S_{i}\right)\right)>\varepsilon-\eta$.

Since if we replace $\eta$ by $\eta^{\prime} \in(0, \eta)$, then the $S_{i}$ are replaced by $S_{i}^{\prime} \subset S_{i}$ and

$$
\operatorname{diam}\left(\frac{1}{n}\left(\sum_{i=1}^{n} S_{i}\right)\right) \geqslant \operatorname{diam}\left(\frac{1}{n}\left(\sum_{i=1}^{n} S_{i}^{\prime}\right)\right)>\varepsilon-\eta^{\prime}
$$

this shows that $\operatorname{diam}\left(\frac{1}{n}\left(\sum_{i=1}^{n} S_{i}\right)\right) \geqslant \varepsilon$.

(3) $\Rightarrow(2)$ : Let $x_{i}, \ldots, x_{n} \in S(X)$, let $\lambda$ and $\alpha$ be two non-negative real numbers, and let $\delta \in(0, \alpha \lambda \varepsilon)$ be fixed. By hypothesis, $B\left(X^{\star}\right)$ does not contain any $\varepsilon$-combination of $w^{\star}$-slices, so there exist $f_{1}, \ldots, f_{n}, g_{1}, \ldots, g_{n}$ so that.

a. for all $i, f_{i} \in S_{i}$ and $g_{i} \in S_{i}$, where $S_{i}=S\left(x_{i}, B\left(X^{\star}\right), \delta\right)$;

b. $\left\|\frac{1}{n} \sum_{i=1}^{n} f_{i}-\frac{1}{n} \sum_{i=1}^{n} g_{i}\right\|>\varepsilon(1-\alpha)$. 
So there exists $u \in S(X)$ such that $\frac{1}{n} \sum_{i=1}^{n}\left(f_{i}-g_{i}\right)(u)>\varepsilon(1-\alpha)$, hence

$$
\begin{aligned}
\frac{1}{n} \sum_{i=1}^{n}\left(\left\|x_{i}+\lambda u\right\|+\left\|x_{i}-\lambda u\right\|\right) & \geqslant \frac{1}{n} \sum_{i=1}^{n}\left(f_{i}\left(x_{i}+\lambda u\right)+g_{i}\left(x_{i}-\lambda u\right)\right) \\
& \geqslant \frac{1}{n} \sum_{i=1}^{n} f_{i}\left(x_{i}\right)+\frac{1}{n} \sum_{i=1}^{n} g_{i}\left(x_{i}\right)+\lambda\left(\frac{1}{n} \sum_{i=1}^{n}\left(f_{i}-g_{i}\right)(u)\right) \\
& \geqslant 1-\delta+1-\delta+\lambda \varepsilon(1-\alpha) \\
& \geqslant 2-2 \lambda \alpha \varepsilon+\lambda \varepsilon(1-\alpha) \\
& \geqslant 2+\lambda \varepsilon(1-3 \alpha) .
\end{aligned}
$$

So we have:

$$
\frac{1}{n} \sum_{i=1}^{n}\left(\frac{\left\|x_{i}+\lambda u\right\|+\left\|x_{i}-\lambda u\right\|-2}{\lambda}\right) \geqslant \varepsilon(1-3 \alpha) .
$$

This shows that, for every $\lambda>0$ :

$$
\sup _{y \in X,\|y\|=\lambda} \frac{1}{n} \sum_{i=1}^{n}\left(\frac{\left\|x_{i}+y\right\|+\left\|x_{i}-y\right\|-2}{\|y\|}\right) \geqslant \varepsilon
$$

whence

$$
\limsup _{y \in X,\|y\| \rightarrow 0} \frac{1}{n} \sum_{i=1}^{n}\left(\frac{\left\|x_{i}+y\right\|+\left\|x_{i}-y\right\|-2}{\|y\|}\right) \geqslant \varepsilon .
$$

$(2) \Rightarrow(1):$ Let $x_{i}, \ldots, x_{n} \in S(X)$ and $\eta \in(0,1)$. By hypothesis, there exists $u \in S(X)$ and $t \in(0, \eta / 3)$ such that

$$
\frac{1}{n} \sum_{i=1}^{n}\left(\frac{\left\|x_{i}+t u\right\|+\left\|x_{i}-t u\right\|-2}{t}\right) \geqslant \varepsilon-\frac{\eta}{3}
$$

Since the real functions $\varphi_{i}: t \rightarrow\left\|x_{i}+t u\right\|$ are convex, if $\varphi_{i}^{\prime}$ denotes the right derivative of $\varphi_{i}$, we have, for $t>0$ :

$$
\varphi_{i}^{\prime}(t) \geqslant \frac{1}{t}\left(\varphi_{i}(t)-\varphi_{i}(0)\right) \text { and } \varphi_{i}^{\prime}(-t) \leqslant \frac{1}{t}\left(\varphi_{i}(0)-\varphi_{i}(-t)\right)
$$

So

$$
d^{+}\left\|x_{i}+t u\right\|(u) \geqslant \frac{1}{t}\left(\left\|x_{i}+t u\right\|-1\right)
$$

and

$$
-d^{+}\left\|x_{i}-t u\right\|(u) \geqslant \frac{1}{t}\left(\left\|x_{i}-t u\right\|-1\right) .
$$


Summing these inequalities for $1 \leqslant i \leqslant n$ :

$$
\sum_{i=1}^{n}\left(d^{+}\left\|x_{i}+t u\right\|-d^{+}\left\|x_{i}-t u\right\|\right)(u) \geqslant \sum_{i=1}^{n} \frac{\left\|x_{i}+t u\right\|+\left\|x_{i}-t u\right\|-2}{t} .
$$

Therefore, using (1):

$$
\frac{1}{n} \sum_{i=1}^{n}\left(d^{+}\left\|x_{i}+t u\right\|-d^{+}\left\|x_{i}-t u\right\|(u)\right) \geqslant \varepsilon-\frac{\eta}{3}
$$

Putting $y_{i}=\frac{x_{i}+t u}{\left\|x_{i}+t u\right\|}, z_{i}=\frac{x_{i}-t u}{\left\|x_{i}-t u\right\|}$ we have that

(a) For all $i, y_{i}, z_{i} \in S(X)$.

(b) For all $i,\left\|x_{i}-y_{i}\right\| \leqslant\left|\frac{\left\|x_{i}+t u\right\|-1}{\left\|x_{i}-t u\right\|}\right|+\frac{\|t u\|}{\left\|x_{i}+t u\right\|} \leqslant \frac{2 t}{\left\|x_{i}+t u\right\|} \leqslant 3 t$ so $\left\|x_{i}-y_{i}\right\|<\eta$ and similarly $\left\|x_{i}-z_{i}\right\|<\eta$.

(c) $\frac{1}{n} \sum_{i=1}^{n}\left(d^{+}\left\|y_{i}\right\|-d^{+}\left\|z_{i}\right\|\right)(u) \geqslant \varepsilon-\eta$.

Let us check condition (c). Indeed, for each $i$,

$$
\left\|x_{i}+t u\right\| d^{+}\left\|y_{i}\right\|(u)=d^{+}\left\|x_{i}+t u\right\|(u)
$$

and

$$
\left\|x_{i}-t u\right\| d^{+}\left\|z_{i}\right\|(u)=d^{+}\left\|x_{i}-t u\right\|(u)
$$

therefore

$$
\begin{aligned}
d^{+}\left\|y_{i}\right\|(u)-d^{+}\left\|z_{i}\right\|(u) \geqslant d^{+} & \left\|x_{i}+t u\right\|(u)-d^{+}\left\|x_{i}-t u\right\|(u) \\
& -\left|\left(\left\|x_{i}+t u\right\|-1\right) d^{+}\left\|x_{i}+t u\right\|(u)\right| \\
& -\left|\left(\left\|x_{i}-t u\right\|-1\right) d^{+}\left\|x_{i}-t u\right\|(u)\right| \\
\geqslant & d^{+}\left\|x_{i}+t u\right\|(u)-d^{+}\left\|x_{i}-t u\right\|(u)-2 t .
\end{aligned}
$$

Condition (c) is obtained by summing these inequalities and applying (2). This shows that the norm of $X$ is $\varepsilon$-average rough and completes the proof of Theorem 1 .

The proof of Theorem 2 is similar and left to the interested reader. Note that in the proof of Theorem $2,(1) \Rightarrow(3)$, it is enough to choose $f_{i}, g_{i} \in S(X)$ satisfying $f_{i}\left(y_{i}\right)>1-\left(\eta-\left\|x_{i}-y_{i}\right\|\right), g_{i}\left(z_{i}\right)>1-\left(\eta-\left\|z_{i}-x_{i}\right\|\right)$ and analogous conditions for $f_{i}(u)$ and $g_{i}(u)$, and to apply the local reflexivity principle ([10, Theorem 3.1, p.33]). 


\section{EXAMPLES AND APPLICATIONS}

In [3], Godefroy and Maurey define a norm \|\| on $X$ to be everywhere octahedral if, for every finite dimensional subspace $Y$ of $X$ and every $\varepsilon>0$, there is an $x \in X \backslash\{0\}$ (depending on $Y$ and $\varepsilon$ ) such that for all $t \in Y, \quad\|t+x\| \geqslant(1-\varepsilon)(\|t\|+\|x\|)$.

EXAMPLE: The usual norm in $\ell_{1}(N)$ is everywhere octahedral. Indeed, let $Y$ be a finite dimensional supspace of $\ell_{1}(\mathrm{~N})$ and $\varepsilon>0$. By compactness of $S(Y)$, we can find an $n \in \mathrm{N}$ such that, if $Z$ is the subspace of $\ell_{1}(\mathrm{~N})$ whose elements are supported by the $n$ first coordinates, then for all $y \in S(Y)$,

$$
d(y, Z)=\inf \{\|y-z\| ; z \in Z\}<\varepsilon / 2 .
$$

Let $x \in \ell^{1}(\mathrm{~N}) \backslash\{0\}$ have its first $n$ coordinates equal to 0 . If $y \in Y$, choose $z \in Z$ such that $\|y-z\|<\frac{e}{2}\|y\|$, then

$$
\begin{aligned}
\|y+x\| & \geqslant\|z+x\|-\|y-z\| \\
& \geqslant\|z\|+\|x\|-\|y-z\| \\
& \geqslant\|y\|+\|x\|-2\|y-z\| \\
& \geqslant(1-\varepsilon)(\|y\|+\|x\|)
\end{aligned}
$$

as required.

Proposition 3. An everywhere octahedral norm is 2 -average rough.

Proof: Observe that by homogeneity, a norm on $X$ is everywhere octahedral if and only if, for every finite dimensional subspace $Y$ of $X$ and every $\varepsilon>0$, there is an $x \in X, \quad\|x\|=1$ such that, for all $t \in Y$ and $\alpha \in \mathbf{R}, \quad\|t+\alpha x\| \geqslant(1-\varepsilon)(\|t\|+|\alpha|)$.

Let $\varepsilon>0$ and let $x_{1}, \ldots, x_{n} \in X$ be of norm 1 . Denote by $Y$ the linear space spannned by $x_{1}, \ldots, x_{n}$. By the previous remark, there is an $x \in S(X)$ such that for all $y \in Y$,

$$
\|t+\sqrt{\varepsilon} x\| \geqslant(1-\varepsilon)(\|t\|+\sqrt{\varepsilon})
$$

and

$$
\|t-\sqrt{\varepsilon} x\| \geqslant(1-\varepsilon)(\|t\|+\sqrt{\varepsilon}) .
$$

So

$$
\frac{\|t+\sqrt{\varepsilon} x\|+\|t-\sqrt{\varepsilon} x\|-2\|t\|}{\sqrt{\varepsilon}} \geqslant 2(1-\varepsilon-\sqrt{\varepsilon}\|t\|) .
$$

Applying this inequality successively for $t=x_{i}, \quad 1 \leqslant i \leqslant n$, and summing:

$$
\frac{1}{n} \sum_{i=1}^{n} \frac{\left\|x_{i}+h_{\varepsilon}\right\|+\left\|x_{i}-h_{\varepsilon}\right\|-2}{\left\|h_{e}\right\|} \geqslant 2(1-\varepsilon-\sqrt{\varepsilon}),
$$


where $h_{\varepsilon}=\sqrt{\varepsilon} x$. This inequality holds for arbitary $\varepsilon>0$, so the proposition follows from Theorem 2(2).

REMARKs: (a) The usual norm in $\ell_{1}(\mathrm{~N})$ is everywhere octahedral, hence, by Proposition 3 , it is 2 -average rough.

(b) Remark (a) is false if we replace the usual norm of $\ell^{1}(N)$ by an equivalent norm. Indeed, there exists on $c_{0}(\mathrm{~N})$ an equivalent locally uniformly rotund norm, hence its unit ball is dentable and the dual norm in $\ell^{1}(N)$ is not even rough.

(c) The author does not know if the converse of Proposition 3 holds.

Proposition 4. Let $X$ be a separable Banach space. The.following are equivalent:

1. $X$ has an equivalent $\varepsilon$-average rough norm for some $\varepsilon>0$;

2. $X$ has an equivalent 2 -average rough norm;

3. $X$ contains $\ell^{1}(\mathrm{~N})$.

Proof: $(1) \Rightarrow(3)$ : If there exists an $\varepsilon$-average norm on $X$, then, by Theorem 1 , $B\left(X^{\star}\right)$ does not contain any $\varepsilon$-combination of $w^{\star}$-slices, and so, by a result of Bourgain ([1, lemme $3-7]), X$ contains $\ell^{1}(N)$.

$(3) \Rightarrow(2)$ : If $X$ is separable and contains $\ell^{1}(N)$, then Godefroy and Maurey ([3, corollaire III.13]) showed that there exists on $X$ an everywhere octahedral norm and by Proposition 3 , this norm is 2 -average rough.

$(2) \Rightarrow(1)$ is obvious.

Proposition 5. If a Banach space $X$ is $\varepsilon$-average rough, then there is a separable closed subspace $Y \subset X$ such that $Y$, with the restricted norm of $X$, is also average rough.

Proof: Let $Y_{0}$ be a one-dimensional subspace of $X$ and let us define a sequence of finite dimensional subspaces of $X$ in the following way: suppose $Y_{0}, Y_{1}, \ldots, Y_{p}$ have been defined and choose a $\frac{1}{p}$-net $\mathcal{A}_{p}=\left\{x_{1}, x_{2}, \ldots, x_{k_{p}}\right\}$ in $S\left(Y_{p}\right)$ and for each subset $A$ of $\mathcal{A}_{p}$, if we denote $A=\left\{t_{1}, \ldots, t_{n}\right\}$, choose $y_{1}^{A}, \ldots, y_{n}^{A}, z_{1}^{A}, \ldots, z_{n}^{A}$ and $u^{A} \in S(X)$ satisfying:

a. for all $i \in\{1, \ldots, n\}, \quad\left\|y_{i}^{A}-t_{i}\right\|<\frac{1}{p}$ and $\left\|z_{i}^{A}-t_{i}\right\|<\frac{1}{p}$;

b. $\frac{1}{n} \sum_{i=1}^{n}\left(d^{+}\left\|y_{i}^{A}\right\|-d^{+}\left\|z_{i}^{A}\right\|\right)\left(u^{A}\right)>\varepsilon-\frac{1}{p}$.

Let $Y_{p+1}$ be the subspace of $X$ spanned by $Y_{p}$ and all the $y_{i}^{A}, z_{i}^{A}, u^{A}$ (for all $\left.A \subset \mathcal{A}_{p}\right)$. Let $Y=\overline{\bigcup_{p \in N} Y_{p}}$. Then $Y$ is a separable subspace of $X$ and $Y$ is $\varepsilon$-average rough. 
COROLLARY 6. Let $X$ be a Banach space (not necessarily separable) which is $\varepsilon$-average rough for some $\varepsilon>0$. Then $X$ contains $\ell^{1}(\mathrm{~N})$.

Proof: By Proposition 5, there exists a separable subspace $Y$ of $X$ which is $\varepsilon$-average rough. By Proposition $4, Y$ contains $\ell^{1}(N)$ and the corollary follows.

\section{REFERENCES}

[1] J. Bourgain, 'La propriété de Radon Nikodym', Publications de l'université Pierre et Marie Curie 36 (1979).

[2] N. Goussoub, G. Godefroy, B. Maurey and W. Schachermayer, 'Some topological and geometrical structures in Banach spaces', Mem. Amer. Math. Soc. (to appear).

[3] G. Godefroy and B. Maurey, 'Normes lisses et normes anguleuses sur les espaces de Banach séparables' (to appear).

[4] G. Godini, 'Rough and strongly rough norms on Banach spaces', Proc. Amer. Math. Soc. 87 (1983), 239-245.

[5] K. John and V. Zizler, 'On rough norms on Banach spaces', Comment. Math. Univ. Carolin. 18 (1978), 335-349.

[6] E.B. Leach and J.H.M Whitfield, 'Differentiable functions and rough norms on Banach spaces', Proc. Amer. Math. Soc. 33 (1972), 120-126.

[7] H.P. Rosenthal, 'On the structure of non-dentable closed bounded convex sets' (to appear).

[8] F. Sullivan, 'Dentability, smoothability and stronger properties in Banach spaces', Indiana Univ. Math. J. 26 (1977), 545-553.

[9] J. Whitfield and V. Zizler, 'A survey of rough norms with applications', Contemp. Math. 54 (1986).

[10] D. Van Dulst, 'Reflexive and superreflexive Banach spaces', Mathematical Centre Tracts 102, Amsterdam 1978.

Equipe d'Analyse fonctionelle

Université Paris VI

FRANCE 